Joachim Wiemeyer ${ }^{1}$

Ruhr-Universität Bochum

\title{
Die Überwindung der gesellschaftlichen Exklusion als Verpflichtung für die Soziallehre der Kirche
}

Die Soziallehre der Kirche ist im 19. Jh. entstanden, als die neue soziale Gruppe der Industriearbeiter um ihren „Standort" und ihre Anerkennung und Teilhabe in der Gesellschaft kämpfte. Im Kontext des damaligen ständestaatlichen Denkens ging es um die „Standwerdung der Arbeiterschaft" zur Integration dieser neuen sozialen Großgruppe in die Gesellschaft. Zu dieser Inklusion hat die Soziallehre der Kirche maßgeblich beigetragen. In westlichen Industrieländern war Mitte der 1960er Jahre diese gesellschaftliche Teilhabe nach der Verleihung des Wahlrechts (1919 in Deutschland), einen hohen gewerkschaftlichen Organisationsgrad, Mitbestimmungsrechte in Betrieben und Unternehmen, soziale Absicherung und Vermögensbildung in

1 Joachim Wiemeyer - Prof. Dr. lic. theol. Promotion in Volkswirtschaftslehre, Habilitation in Christliche Sozialwissenschaften an der Universität Münster, Prof. für Christliche Gesellschaftslehre an der Kath.- Theologischen Fakultät der RuhrUniversität Bochum. Berater der Caritaskommission der Deutschen Bischofskonferenz, Mitglied im Ethikbeirat der Pax-Bank Köln. Forschungsschwerpunkte: Wirtschafts- und Unternehmensethik, Umweltethik, Wohlfahrtsverbände. 
Arbeitnehmerhand weitgehend gelungen. Dies sah und sieht bis heute in Schwellen- und Entwicklungsländern anders aus.

Angeregt durch das Zweite Vatikanische Konzil, vor allem die 1965 verabschiedete Pastoralkonstitution Gaudium et spes und die Entwicklungsenzyklika Populorum progressio von Paul VI., (1967) hatte sich die 2. Vollversammlung der lateinamerikanischen Bischöfe 1968 in Medellín (Kolumbien) den Armen ihres Kontinents zugewandt. Die lateinamerikanische „Theologie der Befreiung“ hatte dazu die Grundlagen gelegt. Die späteren Vollversammlungen des lateinamerikanischen Episkopats in Puebla 1979 und Santo Domingo 1992 haben diese „Option“ unterstrichen und durch die Option für die Jugend ergänzt. Zwar gehört die Zuwendung zu den Armen zu den biblisch fundierten Grundlagen des Christentums (LK 4,18; Mt 25,16-31), aber so zentral wie in den Dokumenten der lateinamerikanischen Kirche ist diese Dimension früher nicht herausgestrichen worden. Sie hat ausgestrahlt auf die Lehrverkündigungen anderer Ortskirchen wie der US-amerikanischen Bischofskonferenz mit ihrem Wirtschaftshirtenbrief von 1986, Nr. 86-88 und dem dadurch angeregten ökumenischen Sozialwort der Deutschen Bischöfen zusammen mit der EKD zur wirtschaftlichen und sozialen Lage in Deutschland 1997, Nr. 105-107. In den USA und Deutschland reagierte man damit auf wachsende soziale Probleme wie das der Langzeitarbeitslosigkeit. Auch Johannes Paul II. hat die Formel der „vorrangigen Option für die Armen" in Sollicitudo rei socialis 1987, Nr. 42 und Centesimo anno 1991, Nr. 11 aufgegriffen. ${ }^{2}$

In der Kirche Lateinamerikas ist die „Option für die Armen“ durch eine genauere Betrachtung von Armutsursachen und Armutsgruppen weiterentwickelt worden. Dabei wurde festgestellt, dass größere Gruppen der Gesellschaft nicht einfach arm sind. Dies könnte ja auch ein unvermeidbares Schicksal oder durch die Armen selbstverschuldet sein, sondern gesellschaftliche Mechanismen, Machtverhältnisse und ökonomische Strukturen sowie einflussreiche Wertvorstellungen (ethnische Vorurteile) bedingen Armut und verursachen einen längerfristigen bzw. dauerhaften

2 Vgl. auch Päpstlicher Rat für Gerechtigkeit und Frieden, Kompendium der Soziallehre der Kirche, Freiburg i Br. 2006, Nr. 182-184. 
Verbleib von Menschen in der Armut. Deshalb soll mit der aus bestimmten Ansätzen der Soziologie stammenden Begrifflichkeit „Exklusion“ von der gesellschaftlichen Teilhabe bzw. der „Ausgeschlossenen“ bzw. der „Ausgegrenzten“ auf diese Problematik hingewiesen werden. In das Dokument von Aparecida, der letzten Vollversammlung der lateinamerikanischen Bischöfe $2007^{3}$, ist diese Perspektive der Exklusion ausdrücklich eingeflossen. Maßgeblich an der Erstellung des Dokuments als Leiter der Redaktionskommission war der damalige Erzbischof von Buenos Aires Jorge Mario Bergoglio beteiligt. Daher verwundert es nicht, dass er als Papst Franziskus diesen Gedanken des gesellschaftlichen Ausschlusses großer Gruppen der Gesellschaft immer wieder aufgreift und in seine Verkündigung einbezieht.

Im Folgenden wird zunächst in einem ersten Abschnitt der soziale Ausschluss anhand der Dokumente von Aparecida sowie der Verkündung von Papst Franziskus vor allem in Evangelii Gaudium und Laudato Si' geschildert. Im zweiten Abschnitt erfolgt dann eine systematische Reflexion aus der Sicht der Christlichen Sozialethik.

\section{Die „Ausgeschlossenen“ in der Sozialverkündigung der Kirche}

In Lateinamerika bildeten europäische Einwanderer seit der spanischen bzw. portugiesischen Eroberungen die Oberschicht. Bei ihnen konzentrierte sich die politische und ökonomische Macht. Dies kommt besonders in der Bodenverteilung vieler Ländern zum Ausdruck, die durch Großgrundbesitz gekennzeichnet ist. Eine schmale Schicht verfügt über die Masse des Bodens. Die Forderung nach einer Agrarreform zur Neuverteilung des Bodens, wie sie in Gaudium et spes Nr. 71 sowie in Populorum progressio Nr. 24 erhoben wurde, blieb unwirksam, so dass Benedikt XVI. dies gut 50 Jahre später in Caritas in veritate 2009, Nr. 27 erneut anmahnen musste.

3 Schlussdokument der 5. Generalversammlung des Episkopats von Lateinamerika und der Karibik (Stimmen der Weltkirche Nr. 41), Hrsg. Sekretariat der Deutschen Bischofskonferenz, Bonn 2007. 
Systematisch wurden und werden die Ureinwohner sowie die aus Afrika importierten Sklaven und ihre Nachkommen diskriminiert. ${ }^{4}$ Der verbreitete „Machismo“ beinhaltet eine Dominanz der Männer über Frauen. Lateinamerika ist die Weltregion, in der die sozialen Unterschiede zwischen „arm“ und „reich“ am größten sind, weil ökonomische Ungleichheit durch die ethnischen Ungleichheiten noch verschärft wird.

In dem Dokument von Aparecida (Nr. 65) ${ }^{5}$ werden diese und weitere Gruppen, die am Rande der Gesellschaft stehen und ausgeschlossen sind, im Einzelnen benannt:

„Zu ihnen gehören: die indigenen und afroamerikanischen Gemeinschaften, die vielfach weder in ihrer Würde anerkannt noch mit gleichen Lebenschancen ausgestattet werden; viele Frauen, die wegen ihres Geschlechts, ihrer Rasse oder ihrer wirtschaftlich-sozialen Lage aus der Gesellschaft ausgeschlossen werden; Jugendliche, die nur unzureichend ausgebildet werden, keine Gelegenheit haben, weiter zu studieren, und auch keinen Zugang zum Arbeitsmarkt erhalten, um sich zu entfalten und eine Familie gründen zu können; viele Arme, Arbeitslose, Migranten, Zwangsumgesiedelte, Bauern ohne Land, die in der informellen Wirtschaft zu überleben suchen; Jungen und Mädchen, die der Kinderprostitution ausgesetzt sind, die nicht selten mit dem Sextourismus verbunden ist; aber auch die Kinder, die Opfer von Abtreibungen wurden. Millionen Menschen und Familien leben im Elend und hungern sogar. Wir sind auch besorgt um alle, die drogenabhängig sind, um Menschen mit Behinderungen, Opfer und Träger schwerer Krankheiten wie Malaria, Tuberkulose, HIV / AIDS, die sich vom Zusammenleben in Familie und Gesellschaft ausgeschlossen fühlen und an Einsamkeit leiden. Wir übersehen auch nicht die Opfer von Entführungen, von Gewalt und Terrorismus, die Opfer bewaffneter Konflikte und der Unsicherheit in den Städten. Auch die alten Menschen,

4 Vgl. zur Ausgrenzung von Minderheiten: R. A. Tuazon, Kulturelle Minderheiten und die katholische Soziallehre, „Concilium“ 53. Jg. (2017), S. 274-283.

5 Vgl. dazu N. Mette, „Überflüssig und menschlicher Abfall“ (Dokument Aparecida 65), Soziale Exklusion-eine himmelschreiende Ungerechtigkeit, „Pastoraltheologische Informationen“ 32. Jg. Heft 1, S. 197-220. 
die sich nicht nur aus dem Produktionssystem ausgeschlossen fühlen, sondern häufig auch von ihren Familien als störend und lästig angesehen werden. Schließlich schmerzt uns die unmenschliche Lage, der die meisten Gefangenen ausgesetzt sind. Auch sie brauchen unsere solidarische Zuwendung und unsere geschwisterliche Hilfe. Eine Globalisierung ohne Solidarität wirkt sich negativ auf die ärmsten Schichten aus. Dabei geht es nicht allein um Unterdrückung und Ausbeutung, sondern um etwas Neues, um den gesellschaftlichen Ausschluss. Durch ihn wird die Zugehörigkeit zur Gesellschaft, in der man lebt, untergraben, denn man lebt nicht nur unten, oder am Rande bzw. ohne Einfluss, sondern man steht draußen. Die Ausgeschlossenen sind nicht nur "Ausgebeutete«, sondern »Überflüssige« und »menschlicher Abfall«."

Der Hinweis im letzten Satz auf „Überflüssige“ und „menschlichen Abfall" kennzeichnet den Extremfall sozialer Exklusion. Während „Ausgebeutete" in den ökonomischen Kreislauf einbezogenen sind und dort eine Funktion erfüllen, wenn auch unter ungerechten Bedingungen, weil ihnen ein Teil des von ihnen Erwirtschafteten vorenthalten wird, sind „Überflüssige“ oder „menschlicher Abfall“ nicht in gesellschaftliche Prozesse und Funktionsmechanismen einbezogen. Es handelt sich u.a. um Menschen, die am Rande einer Müllkippe leben und deren Leben darin besteht, Müllberge nach noch brauchbarem zu durchsuchen. Diese Menschen werden von der Gesellschaft nicht benötigt und werden von anderen als „überflüssig“ oder als „menschlicher Abfall“ angesehen. Solche Zustände widerstreiten der Botschaft Jesu:

„In besonderer Weise finden wir Jesus auch in den Armen, Bedrückten und Kranken (Mt 25,37-40), die unseren Einsatz fordern und uns mit ihrem Glauben, ihrer Geduld im Leiden und ihrem ständigen Überlebenskampf ein Beispiel sind. Wie oft sind es gerade die Armen und Leidenden, die uns das Evangelium nahebringen! In ihnen Jesus Christus zu erkennen und zu begegnen sowie die Rechte der Ausgeschlossenen zu verteidigen - daran misst die Kirche ihre Treue zu Jesus Christus. Jesus Christus in den Armen zu begegnen, gehört zum Kern unseres Glaubens an Jesus Christus. Indem wir sein Leidensantlitz auf dem ihren wieder erkennen und ihm in den Bedrückten und Ausgegrenzten begegnen, deren unendliche Würde er selbst uns offenbart, entwickelt sich unsere Option für die 
Armen. Die Nachfolge Jesu Christi lässt uns Freundschaft mit den Armen schließen und macht uns mit ihrem Schicksal solidarisch“ (Nr. 257).

Die Bischofsversammlung (Nr. 402) verpflichtet sich, diese Gruppen ins Zentrum der Sozialpastoral der Kirche zu stellen.

Das Apostolische Schreiben Evangelii Gaudium (2013), das als Antrittsenzyklika von Papst Franziskus gewertet werden kann, hat besonders in seinen wirtschaftskritischen Passagen hohe Aufmerksamkeit erregt $^{6}$, aber auch Widerspruch hervorgerufen. Dies gilt vor allem für folgenden Absatz:

„Ebenso wie das Gebot, du sollst nicht töten“ eine deutliche Grenze setzt, um den Wert des menschlichen Lebens zu sichern, müssen wir heute ein ,Nein zu einer Wirtschaft der Ausschließung und der Disparität der Einkommen' sagen. Diese Wirtschaft tötet. Es ist unglaublich, dass es kein Aufsehen erregt, wenn ein alter Mann, der gezwungen ist, auf der Straße zu leben, erfriert, während eine Baisse um zwei Punkte in der Börse Schlagzeilen macht. Das ist Ausschließung. Es ist nicht mehr zu tolerieren, dass Nahrungsmittel weggeworfen werden, während es Menschen gibt, die Hunger leiden. Das ist soziale Ungleichheit. Heute spielt sich alles nach den Kriterien der Konkurrenzfähigkeit und nach dem Gesetz des Stärkeren ab, wo der Mächtigere den Schwächeren zunichtemacht. Als Folge dieser Situation sehen sich große Massen der Bevölkerung ausgeschlossen und an den Rand gedrängt: ohne Arbeit, ohne Aussichten, ohne Ausweg. Der Mensch an sich wird wie ein Konsumgut betrachtet, das man gebrauchen und dann wegwerfen kann. Wir haben die ,Wegwerfkultur' eingeführt, die sogar gefördert wird. Es geht nicht mehr einfach um das Phänomen der Ausbeutung und der Unterdrückung, sondern um etwas Neues: Mit der Ausschließung ist die Zugehörigkeit zu der Gesellschaft, in der man lebt, an ihrer Wurzel getroffen, denn durch sie befindet man sich nicht in der Unterschicht, am Rande oder gehört zu den Machtlosen, sondern man steht draußen. Die Ausgeschlossenen sind nicht ,Ausgebeutete', sondern Müll, ,Abfall‘. “

6 Vgl. J. Wiemeyer, Papst Franziskus und die Wirtschaft, „Amosinternational. Gesellschaft gerecht gestalten" 8 (2014) 1, S. 50-53 und derselbe, Evangelii Gaudium - das Programm eines Pontifikats, „Die neue Ordnung“ 68 (2014) 2, S. 100-109. 
Papst Franziskus bestreitet die wirtschaftsliberale These, dass von Wirtschaftswachstum letztlich alle Glieder der Gesellschaft profitieren würden, weil der Wohlstand durch die verschiedenen Einkommensschichten durchsickert (vgl. EG Nr. 53). Extremer Konsum der Reichen führt zur Vergeudung, z. B. der Vernichtung von Nahrungsmitteln, während andere Hunger leiden. Vielfach sind herrschende Gruppen korrupt, die sich ungerecht bereichern, was nur den Zorn der Ausgegrenzten steigern kann: „Das wird noch anstößiger, wenn die Ausgeschlossenen jenen gesellschaftlichen Krebs wachsen sehen, der die in vielen Ländern - in den Regierungen, im Unternehmertum und in den Institutionen - tief verwurzelte Korruption ist, unabhängig von der politischen Ideologie der Regierenden." (Nr. 60)

Die Folgen extremer Ungerechtigkeiten sind Krieg und Gewalt:

„Wenn die lokale, nationale oder weltweite Gesellschaft einen Teil ihrer selbst in den Randgebieten seinem Schicksal überlässt, wird es keine politischen Programme, noch Ordnungskräfte oder Intelligence geben, die unbeschränkt die Ruhe gewährleisten können. Das geschieht nicht nur, weil die soziale Ungleichheit gewaltsame Reaktionen derer provoziert, die vom System ausgeschlossen sind, sondern weil das gesellschaftliche und wirtschaftliche System an der Wurzel ungerecht ist. Wie das Gute dazu neigt, sich auszubreiten, so neigt das Böse, dem man einwilligt, das heißt die Ungerechtigkeit, dazu, ihre schädigende Kraft auszudehnen und im Stillen die Grundlagen jeden politischen und sozialen Systems aus den Angeln zu heben, so gefestigt es auch erscheinen mag. Wenn jede Tat ihre Folgen hat, dann enthält ein in den Strukturen einer Gesellschaft eingenistetes Böses immer ein Potenzial der Auflösung und des Todes.“ (Nr. 202).

Im Rahmen seiner Umweltenzyklika Laudato si' (2015) greift Papst Franziskus die Problematik auf, wenn er die soziale Spaltung der Städte anspricht:

„In einigen ländlichen und städtischen Zonen hat die Privatisierung von Geländen dazu geführt, dass der Zugang der Bürger zu Gebieten von besonderer Schönheit schwierig wird. Unter anderem werden ,ökologische“ Wohnanlagen geschaffen, die nur einigen wenigen dienen, wo man zu vermeiden sucht, dass andere eintreten und die künstliche 
Ruhe stören. Eine schöne Stadt voller gut gepflegter Grünflächen findet man gewöhnlich in einigen ,sicheren' Gebieten, jedoch kaum in weniger sichtbaren Zonen, wo die von der Gesellschaft Ausgeschlossenen leben.“ (Nr. 45).

In weltweiten Konferenzen wird zwar das Schicksal der Armen behandelt, steht aber nicht, wie es sein müsste, im Zentrum:

„Ich möchte darauf hinweisen, dass man gewöhnlich keine klare Vorstellung von den Problemen hat, die besonders die Ausgeschlossenen heimsuchen. Sie sind der größte Teil des Planeten, Milliarden von Menschen. Heute kommen sie in den internationalen politischen und wirtschaftlichen Debatten vor, doch oft scheint es, dass ihre Probleme gleichsam als ein Anhängsel angegangen werden, wie eine Frage, die man fast pflichtgemäß oder ganz am Rande anfügt, wenn man sie nicht als bloßen Kollateralschaden betrachtet." (Nr. 49).

„In der gegenwärtigen Situation der globalen Gesellschaft, in der es so viel soziale Ungerechtigkeit gibt und immer mehr Menschen ausgeschlossen und ihrer grundlegenden Menschenrechte beraubt werden, verwandelt sich das Prinzip des Gemeinwohls als logische und unvermeidliche Konsequenz unmittelbar in einen Appell zur Solidarität und in eine vorrangige Option für die Ärmsten. Diese Option bedeutet, die Konsequenzen aus der gemeinsamen Bestimmung der Güter der Erde zu ziehen, doch - wie ich im Apostolischen Schreiben Evangelii gaudium auszuführen versuchte - verlangt sie vor allem, sich die unermessliche Würde des Armen im Licht der tiefsten Glaubensüberzeugungen vor Augen zu führen." (Nr. 158).

Wenn man diese Aussagen näher analysiert, muss man eine angemessene Methode zur Interpretation heranziehen. So lassen sich in der kirchlichen Sozialverkündigung mindestens drei verschiedene Aussageformen ${ }^{7}$ unterscheiden: Dies sind erstens grundsätzliche Aussagen über die Würde des Menschen und die Gerechtigkeit des Zusammenlebens, die sich in den Sozialprinzipien wie Personalität, Subsidiarität, Solidarität und Gemeinwohl niederschlagen. Zweitens sind pragmatische Empfehlungen zu finden. Drittens bedienen sich

7 Vgl. W. Weber, Der soziale Lehrauftrag der Kirche, Köln 1975, S. $17 f$. 
Päpste und Bischöfe der prophetischen Sozialkritik. Offensichtlich sind die zitierten Aussagen von Papst Franziskus ${ }^{8}$ als prophetische Sozialkritik einzuordnen. Damit unterscheidet er sich deutlich von seinem Vorgänger Benedikt XVI., der eher grundsätzliche theologische und ethische Überlegungen anstellte. Eine prophetische Sozialkritik hat angesichts von langjährigen Militärdiktaturen und krassen wirtschaftlichen und sozialen Unterschieden in Lateinamerika ihren sozialen Ort, der biographisch auch Papst Franziskus geprägt hat. Daher hat muss man zudem den Erfahrungshintergrund eines Autors immer miteinbeziehen.

\section{Gesellschaftliche Ausgrenzung aus der Sicht der Christlichen Sozialethik}

Aufgabe der Christlichen Sozialethik ist es, sozialstrukturelle Fragen der Gesellschaftsgestaltung auch im Dialog mit anderen Wissenschaften und der praktischen Philosophie systematisch zu reflektieren. Vor einer systematischen normativen Reflexion und dem Aufzeigen von Handlungsoptionen hat sich die Christliche Sozialethik über den genauen Sachverhalt zu vergewissern. Diese Methodik des „Sehen-UrteilenHandelns" wird seit Mater et magistra (Nr. 236) von Johannes XXIII. der Sozialethik empfohlen. ${ }^{9}$ Fragen gesellschaftlicher Exklusion oder Inklusion werden in verschiedenen sozialwissenschaftlichen Theorien verhandelt. Hier werden zwei dieser Theorien aufgegriffen, nämlich die Gesellschaftstheorie des deutschen Soziologen Niklas Luhmann sowie die Theorie kollektiven Handelns des US-amerikanischen Ökonomen Mancur Olson jr.

8 Vgl. J. Wiemeyer, Die Kapitalismuskritik von Papst Franziskus, in: Der Kapitalismus - ein Feindbild für die Kirchen?, Hrsg. S. Wirz, Zürich-Baden-Baden 2018 (im Erscheinen).

9 Vgl. J. Wiemeyer, Keine Freiheit ohne Gerechtigkeit, Freiburg i Br. 2015, S. 40-45. 


\subsection{Zwei Ansätze der Gesellschaftstheorie}

\subsubsection{Die Systemtheorie}

Der Soziologe Niklas Luhmann (1927-1998) ${ }^{10}$ sieht in seiner Systemtheorie moderne Gesellschaften als „ausdifferenzierte Gesellschaften" an, die durch verschiedene zentrale Funktionsbereiche gekennzeichnet sind. Solche Bereiche sind "Politik“, „Wirtschaft", „Recht“, „Wissenschaft“, „Medien“ etc. Diese Bereiche laufen immer nach eigener Logik ab, etwa im Wirtschaftssystem. Dort ist „Zahlen“ oder „Nichtzahlen“ das zentrale Steuerungsinstrument. Im Bereich der Politik spielt hingegen „Macht“ die zentrale Rolle.

Alle Gesellschaftsmitglieder sind in der systemtheoretischen Logik in verschiedene Funktionsbereiche inkludiert. So ist jeder Staatsbürger in der Demokratie mit seinem Stimmrecht in die Politik integriert. Durch die allgemeine Schulpflicht ist jedes Kind und Jugendlicher in das Bildungssystem einbezogen. Die gegen Bezahlung Arbeitenden sind Teil des Wirtschaftssystems, ebenso auch alle Menschen als Konsumenten, da sie über Geld verfügen, das sie an Märkten ausgeben. Dies gilt auch für die Empfänger staatlicher Sozialleistungen. Auch legale Migranten sind in mehrere Funktionszusammenhänge integriert, weil sie arbeiten und konsumieren, das Bildungs- und Gesundheitssystem von ihnen genutzt wird usw.

Selbst sich illegal in einem Land aufhaltende Personen ${ }^{11}$ können nicht als vollständig ausgeschlossen angesehen werden, wenn sie z. B. einer Erwerbsarbeit nachgehen und damit am Wirtschaftssystem teilhaben. Falls „Illegale“ entdeckt werden und zur Ausreise verpflichtet werden bzw. abgeschoben werden sollen, können sie in einem Rechtsstaat Klage dagegen erheben und während des Verfahrens auch Sozialleistungen erhalten.

10 Vgl. N. Luhmann, Inklusion und Exklusion, in: Soziologie und der Mensch, 2. Aufl., Hrsg. N. Luhmann, Wiesbaden 2005, S. 226-251 (Soziologische Aufklärung. 6).

${ }_{11} \mathrm{Vgl}$. A. Fisch, Inklusion von Menschen ohne Aufenthaltsstatus. Lässt sich ein Recht auf Legalisierung für bestimmte, Statuslose begründen?, in: Beteiligung-Inklusion-Integration, Hrsg. C. Eckstein u.a., Münster 2007, S. 189-202 (Forum Sozialethik, 5). 
Luhmann ${ }^{12}$ schildert, dass es in Dritt-Welt-Ländern Konstellationen gibt, in denen ein bestimmter Personenkreis nicht in diese verschiedenen Funktionsbereiche integriert ist, z. B. weil ihre Geburt vom Staat nicht registriert wurde, sie keinen Personalausweis haben, über keine Wohnung mit Adresse verfügen, ihre Kinder nicht eingeschult werden, sie nicht gegen elementare Lebensrisken (Krankheit) versichert sind. Die Gesellschaft bedarf ihrer nicht, weil sie in keine Funktionsbereiche integriert sind. Sie sind die Ausgeschlossenen, weil für die Gesellschaft Überflüssigen. Da in solchen Ländern fast alle Menschen religiös sind, konstatiert Luhmann, dass die Zugehörigkeit zu einer Kirche bzw. religiösen Gemeinschaft wohl die noch am ehesten vorhandene Form gesellschaftlicher Integration darstellen könnte.

\subsubsection{Die Logik kollektiven Handelns}

Mancur Olson jr. (1932-1998) hat sich mit der „Logik kollektiven Handelns " ${ }^{13}$ beschäftigt. Seine Fragestellung ist die, wie sich in einer Gesellschaft Gruppen bilden, um im gesellschaftlichen Zusammenhang ihre Interessen zu definieren und durchzusetzen sowie ihre Stellung gegenüber anderen Gruppen, etwa durch Einflussnahme im politischen Entscheidungsprozess abzusichern. Gut organisierbar sind kleine Gruppen, die homogen sind, aufgrund ähnlicher Sozialisation, eines gleichen Bildungsstandes, ähnliche Interessen haben und in der Lage sind, ein Machtpotential in der Gesellschaft auszuüben, in dem sie Leistungen für die Gesellschaft bereitstellen, auf diese sie nicht verzichten kann. Ein Beispiel wären Ärzte, die an hohen Einkommen Interesse haben. Ärzte können sich leicht organisieren, weil offensichtlich ist, wer zur Gruppe gehört. Forderungen, die Ärzte durchsetzen, kommen nur ihnen zugute. Sie können eine relativ große Geschlossenheit organisieren, indem sie bei Kampfmaßnahmen auf den Zusammenhalt der Gruppe vertrauen, aber auch in der Lage sind, Personen der eigenen Gruppe, die sich

12 Vgl. N. Luhmann, Inklusion und Exklusion, a.a.O., S. $242 \mathrm{f}$.

13 Vgl. M. Olson Jr., Die Logik kollektiven Handelns, Tübingen 1968 (engl. Original 1965). 
unsolidarisch verhalten, zu sanktionieren. Die durchgesetzten Vorteile (z. B. Honoraranhebungen) sind auf ihre Gruppe beschränkt.

Schwieriger organisierbar sind allgemeine Interessen wie diejenigen von Konsumenten und Steuerzahlern. Wenn sich einige für geringe Steuern, z. B. durch effizientere Staatsausgaben oder für einen besseren Konsumentenschutz einsetzen, dann ist der Vorteil, z. B. im Verhältnis zum Gesamteinkommen, gering (man spart wenige Prozent des Einkommens) und die erreichten Vorteile kommen allen zugute, auch denjenigen, die sich bei der Durchsetzung der Forderung nicht engagiert haben, sondern Trittbrettfahrer sind. Es wird ein öffentliches Gut produziert.

Gewerkschaften organisieren üblicherweise große Gruppen von Menschen, die auch heterogene Interessen (unterschiedlicher Qualifikationsstand der Beschäftigten) haben. Daher ist es für sie schwieriger als für kleine Gruppen, ihre Interessen zu bündeln und zu vertreten. Häufig gelingt ihnen dies, indem sie sich nicht nur für Verbesserungen der Arbeitsbedingungen einsetzen, die auch Nichtmitgliedern zugutekommen, sondern auch weitere Güter (z. B. Beratung und rechtliche Vertretung bei Prozessen im Arbeits- und Sozialrecht) nur für ihre Mitglieder anbieten. Olson nennt dies ,selektive Anreize“. Aufgrund der Arbeitsniederlegung haben sie ein Drohpotential gegenüber Arbeitgebern und der Gesellschaft. Ebenso können negative Sanktionen gegenüber unsolidarischen Trittbrettfahrern erfolgen, z. B. in einem Betrieb die Ächtung und Ausgrenzung von Nichtmitglieder der Gewerkschaft durch ihre Kollegen oder andere Formen der Benachteiligung, (z. B. bei der Urlaubsplanung, Beförderung, Einstufung in Gehaltsklassen), wenn hier der gewerkschaftlich organisierte Betriebsrat ein Mitspracherecht hat.

Es gibt aber noch weitere Gruppen in der Gesellschaft, nämlich latente Gruppen, die von ihrer Lebenssituation am stärksten angewiesen wären, auf sich aufmerksam zu machen und ihre Interessen zu artikulieren. Olson bezeichnet diese als „die vergessenen Gruppen - die schweigend leiden." ${ }^{14} \mathrm{Zu}$ solchen Gruppen gehören sexuell missbrauchte Kinder oder von ihren gewalttätigen Partnern drangsalierte Frauen. Hier kennen sich

14 M. Olson Jr., Die Logik kollektiven Handelns, a.a.O., S 160. 
die gleichermaßen Betroffene gar nicht, so dass sie sich nicht organisieren können. Anderen fehlt dazu die Bildung, um soziale Bewegungen Unterprivilegierter zu gründen und ihre Positionen in die Öffentlichkeit zu tragen. Zusätzlich verfügen sie und andere latente Gruppen nicht über die notwendigen ökonomischen Ressourcen, bzw. das Drohpotential, mit dem sie öffentliche Aufmerksamkeit erregen und die Gesellschaft unter Druck setzen können. Vielmehr sind sie mit einem alltäglichen Überlebenskampf beschäftigt, weil sie nur kurzfristig denken und handeln können.

In den USA ist zuerst in Chicago zur Organisation unterprivilegierter Gruppen das „community organizising “15 entwickelt worden. Es besteht darin, dass eine oder mehrere Koordinatoren versuchen, verschiedene Gruppen der Gesellschaft zusammenzubringen, damit sie eine hinreichende Größe erreichen und eine schlagkräftige Organisation entwickeln können, um ihre Forderungen öffentlich zu artikulieren. Die Erzdiözese Chicago hat häufiger das dazu notwendige Kapital bereitgestellt. Barak Obama war als ein solcher „community organizer" tätig, wobei sein Wahlkampfmotto „Yes we can“ auf eine Ermunterung von Armen zum gesellschaftlichen Handeln basierte, die lange bereits resigniert hatten. Häufiger Anknüpfungspunkt dieser Bewegung waren die verschiedenen christlichen Kirchen und religiösen Gemeinschaften, weil sie die wichtigste Form sozialer Organisationen sind, der ärmere Menschen angehören.

Möglichkeiten und Fähigkeiten Menschen zum Einsatz für ihre Interessen zu ermuntern und zu organisieren sind in Schwellen- und Entwicklungsländern noch viel schlechter als in Industrieländern, zumal ihnen häufig keine hochgebildeten und sozial motivierten Anführer von Havard-Absolventen vom Typ Barak Obama und großzügige Spender zur Verfügung stehen. 2007.

15 Vgl. Community Organizing, Menschen verändern ihre Stadt, Hrsg. L. Penta, Hamburg 


\subsection{Gesellschaftliche Inklusion als Forderung der Christlichen Soziallehre}

Zur Tradition der christlichen Ethik gehört es, philosophische Ethiken kritisch zu rezipieren. In der politischen Theorie der Gegenwart spielt das Konzept des Gesellschaftsvertrages eine wichtige Rolle. Auch Papst Franziskus zieht diesen Begriff heran, wenn er sich sozialen Fragen zuwendet. ${ }^{16}$ Es gibt verschiedene Varianten des Gesellschaftsvertrages. Der „fiktive Gesellschaftsvertrag“ bei John Rawls ${ }^{17}$ (1921-2002) steht im Zentrum der politischen Philosophie der letzten Jahrzehnte. Dies ist deshalb der Fall, weil Rawls mit dem „Schleier des Nichtwissens“ den zentralen ethischen Gesichtspunkt der Unparteilichkeit in das Zentrum seiner Überlegungen stellt. Zudem kennt Rawls in seinen Überlegungen zu ökonomischen Verteilungsfragen eine besondere Rücksichtnahme auf die Armen (Differenzprinzip). Wie ist aus einer solchen Sicht „soziale Exklusion“ bzw. die Lage der „Ausgeschlossenen“ zu beurteilen?

Die Idee des Gesellschaftsvertrages will alle Menschen in einem Staat zu Subjekten machen, weil konzeptionell von ihrer Zustimmung die Gesellschaftsordnung abhängt. Die Gerechtigkeit der Sozialordnung soll dadurch gewährleistet sein, dass jeder den grundlegenden Prinzipien des Zusammenlebens zugestimmt hat. Denn, wenn jemand freiwillig einem Vertrag zugestimmt hat, kann ihm kein Unrecht geschehen. Damit steht diese Konzeption diametral dem sozialen Ausschluss entgegen.

Die Grundidee des Gesellschaftsvertrages geht vielmehr von einer Beteiligung aller an der Festlegung der fundamentalen Grundsätze der Gesellschaftsordnung aus. Die Teilhabe an der Zustimmung zu den Verfassungsprinzipien soll sicherstellen, dass die einzelnen Menschen auch umfassend an den Teilsystemen der Gesellschaft beteiligt sind. Die Anforderungen decken sich weitgehend mit der UN-Menschenrechtserklärung von 1948 bzw. weiteren Menschenrechtserklärungen.

16 Vgl. seine Ansprache am 27.5.2017 vor Arbeitern in Genua. http://w2.vatican.va/ content/francesco/de/speeches/2017/may/documents/papa-francesco_20170527_lavoratori-genova.html (6.4.2018).

17 Vgl. J. Rawls, Theorie der Gerechtigkeit, Frankfurt a.M. 1979. 
So hat jeder einzelne Mensch Rechtssubjekt zu sein, in dem seine Grundrechte gewährleistet sind und durch rechtsstaatliche Verfahren vor einem unabhängigen Gericht eingeklagt werden können. Wem zur Prozessführung oder Verteidigung finanzielle Mittel fehlen, dem muss dies durch staatliche Zuschüsse (Prozesskostenhilfe) ermöglicht werden.

In der politischen Sphäre verfügt jeder Erwachsene über ein Wahlrecht, kann sich in Parteien und Verbänden organisieren, darf sich frei informieren sowie eigene Meinungen verbreiten oder demonstrieren. Jeder hat das Recht einer Religionsgemeinschaft anzugehören, aber auch religiösen Gruppen fernzubleiben, seine Religion mit anderen auszuüben und dafür auch über entsprechende Gebäude (Kirchen, Synagogen, Moscheen, Tempel etc.) und Einrichtungen zu verfügen. Damit jeder Mensch in der Gesellschaft befähigt wird, die vielfältigen Rechte und Möglichkeiten wahrzunehmen, ist ein Bildungswesen erforderlich, dass zum einen zumindest in der grundlegenden Bildung kostenfrei ist, zum anderen aber auch eine Verpflichtung zum Schulbesuch einschließt. Neben Bildung sind weitere Voraussetzungen der gesellschaftlichen Teilhabe Gesundheit und Einkommen. Daher müssen alle Bürger Zugang zu vorbeugenden Maßnahmen (z. B. Impfungen) erhalten und zu medizinischen Behandlungen. Einkommen ist zum einen zu gewährleisten durch staatliche Sozialleistungen. Deren Höhe hängt vom allgemeinen Entwicklungsstand eines Landes ab und sollte das durchschnittliche Einkommen nicht weit unterschreiten. Eigenes Einkommen wird durch wirtschaftliche Teilhabe erzielt, sei es durch Selbstversorgung, als Selbständiger oder als Arbeitnehmer. Daher müssen Zugangsmöglichkeiten zum Arbeitsmarkt bestehen, etwa durch eine qualifizierte Berufsausbildung oder ein Studium. Außerdem muss es Möglichkeiten geben, Eigentum zu bilden und Kredit zu erhalten, um ggf. eine selbständige Existenz aufzubauen.

Neben zahlenmäßig kleinen sozialen Randgruppen ${ }^{18}$ wie Obdachlosen gibt es auch in westlichen Industriegesellschaften zwei größere Gruppen

18 Auf solche Gruppen fokussiert primär der ökumenisch ausgerichtete voluminöse Band Kirchen aktiv gegen Armut und Ausgrenzung. Theologische Grundlagen und praktische Ansätze für Diakonie und Gemeinde, Hrsg. J. Eurich u.a., Stuttgart 2011. 
von Menschen, die von Teilbereichen der Gesellschaft ausgeschlossen sind, nämlich zum einen Migranten, d.h. Personen mit fremder Staatsangehörigkeit und Langzeitarbeitslose. Ausländer haben häufig nur ein befristetes Aufenthaltsrecht und sind von staatsbürgerlichen Rechten ausgeschlossen. Da westliche Gesellschaften auf umfassende Inklusion aller Bürger abzielen, die in ihren Ländern dauerhaft leben, eröffnen sie den Erwerb der Staatsangehörigkeit. Wer sich längere Zeit (z. B. 8 Jahre) in einem Land aufhält, sich eingewöhnt hat, die Sprache beherrscht und sich entschlossen hat, dort dauerhaft zu leben, kann einen Antrag auf Erwerb der Staatsangehörigkeit stellen. Die aufnehmende Gesellschaft wird dies von der Bedingung abhängig machen, ob derjenige über hinreichende Kenntnisse des neuen Landes (Geschichte, Verfassung) verfügt, bisher straffrei geblieben ist und den Lebensunterhalt selbst verdienen kann. Umstritten ist die Frage, ob nach einer Einbürgerung die alte Staatsangehörigkeit aufgegeben werden muss.

In den Arbeitsprozess nicht integriert sind Langzeitarbeitslose. Selbst wenn diese durch Sozialleistungen ein Mindesteinkommen erhalten, stellt für Erwachsene und arbeitsfähige Personen die fehlende Teilhabe am Arbeitsmarkt ein Problem dar. Dies gilt für sie individuell, weil in der Regel durch Arbeit ein höheres Einkommen als durch Sozialleistungen zu erzielen ist, die Entfaltung in der Arbeit, die Zeitstruktur für den einzelnen Menschen hohe Bedeutung hat, sowie selbst durch eigene Leistungen erworbenes Einkommen sowohl für das Selbstbewusstsein der Person, als auch für das Ansehen unter den Mitmenschen wichtig ist. Dies gilt aber gesellschaftlich, weil die produktiven Fähigkeiten von Menschen nicht entfaltet und genutzt werden.

Daher ist die Wirtschaft so $\mathrm{zu}$ organisieren, dass eine möglichst hohe Teilhabe aller arbeitsfähigen Personen sichergestellt ist. Dies hat eine Reihe von Voraussetzungen: Dazu gehört eine Bildungspolitik, die dafür sorgt, dass Menschen mit auf dem Arbeitsmarkt benötigten Qualifikationen zur Verfügung stehen. Erforderlich ist weiterhin eine Wirtschaftspolitik, die im Zusammenspiel von Geld-, Fiskal- und Strukturpolitik für eine hohe Nachfrage nach Arbeitskräften sorgt. Weiterhin sind die Tarifvertragsparteien gefordert, Arbeitsbedingungen so auszuhandeln, dass Arbeit und Einkommen auf möglichst viele 
Arbeitende verteilt werden. Die Teilhabemöglichkeiten müssen aber auch von den Arbeitsfähigen ergriffen werden, was regionale oder berufliche Mobilität erforderlich machen kann.

Dort, wo Exklusion sich nicht auf Teilbereiche der Gesellschaft und einen begrenzten Personenkreis der Gesellschaftsmitglieder beschränkt, sondern größere Gruppen, ggf. sogar die Mehrheit der auf einem Territorium lebenden Menschen bezieht oder für manche Personen eine Vielzahl von Gesellschaftsbereichen umfasst, liegt kein Gesellschaftsvertrag vor. Es fehlt ein Verständnis, „Gesellschaft“ als ein Unternehmen aufzufassen, dass eine umfassende Kooperation aller zum gegenseitigen Vorteil darstellt. Häufig ist eine verfehlte antagonistische Betrachtungsweise Ursache des Übels, weil Gesellschaft als ein „NullSummen-Spiel" angesehen wird, nach dem der eine nur gewinnen kann, was andere verlieren.

Die liberale Theorie des Gesellschaftsvertrages hat primär Individuen im Blick, weniger kulturell geprägte Gemeinschaften, die als ethnische Gruppen, religiöse Minderheiten, eigene Sprachgruppen etc. eine eigene kulturelle Herkunft haben und diese Kultur weiterpflegen wollen. Solche kulturellen Rechte müssen für solche Minderheiten garantiert sein. Nach Johannes XXIII., Pacem in terris Nr. 96 „[...] entspricht es vollkommen den Geboten der Gerechtigkeit, wenn die Staatslenker sich tatkräftig bemühen, die Lebensbedingungen der Minderheit zu heben, namentlich in dem, was deren Sprache, Kultur, Herkommen und Gebräuche sowie wirtschaftliche Unternehmungen und Initiativen betrifft." Wenn Minderheiten in einem Staatsgebiet relativ geschlossen leben, kann dies nach dem Subsidiaritätsprinzip durch lokale Selbstverwaltung (kommunal und in Teilstaaten) gewährleistet werden.

\section{Fazit}

Nach christlichem Verständnis sollen alle Menschen ihre Fähigkeiten und Begabungen entfalten können, ihr Leben eigenverantwortlich gestalten können und zum Gemeinwohl in die Gesellschaft beitragen. Dort, wo dies durch Ausbeutung oder gesellschaftlichen 
Ausschluss nicht geschieht, liegt eine kollektive Selbstschädigung vor, weil eine Gesellschaft nicht alle Fähigkeiten und Begabungen ihrer Gesellschaftsmitglieder und die Kooperationsmöglichkeiten untereinander voll ausschöpft. Eine solche kollektive Selbstschädigung kann nur als „Strukturen der Sünde“ (Johannes Paul II., Sollicitudo rei socialis Nr. 36) bezeichnet werden. Die Überwindung solcher Strukturen ist Aufgabe aller Christen. 


\section{Summary}

Die Überwindung der gesellschaftlichen Exklusion als Verpflichtung für die Soziallehre der Kirche

Aufgrund der Erfahrungen in seinem Heimatkontinent Lateinamerika, in dem viele Menschen am Rande der Gesellschaft leben, tritt Papst Franziskus entschieden für die „Ausgeschlossenen“ ein. Diese leben häufig an und von Müllkippen, sind als Staatsbürger nicht registriert, ihre Kinder besuchen keine Schulen etc. Der Beitrag macht deutlich, dass die Soziallehre der Kirche alle Menschen umfassend zu Teilhabern ihrer jeweiligen Gesellschaft in allen Kultursachbereichen (Politik, Wirtschaft, Bildung, Gesundheit) machen will. Vor dem Hintergrund sozialwissenschaftlicher Theorien (Luhmann, Olson) wird er exemplarisch erläutert, dass in westlichen Industriegesellschaften als Großgruppen vor allem Langzeitarbeitslose und Migranten von der vollen Teilhabe an der Gesellschaft ausgeschlossen sind und sich daher die Integrationsanstrengungen vor allem auf diese beiden Personenkreise beziehen muss.

Schlüsselwörter: Soziale Exklusion, Theologie der Befreiung, Papst Franziskus, Soziallehre der Kirche

\section{The Overcoming of the Social Exclusion as an Obligation for the Social Doctrine of the Church}

Based on his experiences in his home continent of Latin America, where many people live on the margins of society, Pope Francis resolutely supports the "excluded". They often live on and from dumps, are not registered as citizens, their children do not attend schools etc. The article makes it clear that the social doctrine of the church wants to make all people comprehensively partakers of their respective societies in all cultural sectors (politics, economy, education, health). Against the background of social science theories (Luhmann, Olson) he is exemplarily explained that in western industrialized societies as large groups especially the longterm unemployed and migrants are excluded from the full participation in society and therefore the integration efforts above all on this must relate to both groups of people.

Keywords: Social exclusion, Theology of Liberation, Pope Francis, Social Doctrine of the Church 


\section{Bibliographie}

Community Organizing. Menschen verändern ihre Stadt, Hrsg. L. Penta, Hamburg 2007.

Fisch A., Inklusion von Menschen ohne Aufenthaltsstatus. Lässt sich ein Recht auf Legalisierung für bestimmte "Statuslose“ begründen?, in: Beteiligung-Inklusion-Integration, Hrsg. Ch. Eckstein u.a., Münster 2007, S. 189-202 (Forum Sozialethik, 5).

Franziskus, Ansprache von Papst Franziskus. Begegnung mit Vertretern der Welt der Arbeit, Internetdokument auf http://w2.vatican.va/content/francesco/de/speeches/2017/may/documents/papa-francesco_20170527_lavoratori-genova.html vom 27.05.2017 (6.4.2018).

Kirchen aktiv gegen Armut und Ausgrenzung. Theologische Grundlagen und praktische Ansätze für Diakonie und Gemeinde, Hrsg. J. Eurich u.a., Stuttgart 2011.

Luhmann N., Inklusion und Exklusion, in: Soziologie und der Mensch, 2. Aufl., Hrsg. N. Luhmann, Wiesbaden 2005, S. 226-251 (Soziologische Aufklärung, 6).

Mette N., „Überflüssig und menschlicher Abfall“ (Dokument Aparecida 65). Soziale Exklusion - eine himmelschreiende Ungerechtigkeit, „Pastoraltheologische Informationen" 32 (2012) Nr 1, S. 197-220.

Olson Jr. M., Die Logik kollektiven Handelns, Tübingen 1968 (engl. Original 1965).

Päpstlicher Rat für Gerechtigkeit und Frieden, Kompendium der Soziallehre der Kirche, Freiburg i Br. 2006.

Rawls J., Eine Theorie der Gerechtigkeit, Frankfurt a.M. 1979.

Schlussdokument der 5. Generalversammlung des Episkopats von Lateinamerika und der Karibik, Hrsg. Sekretariat der Deutschen Bischofskonferenz, Bonn 2007 (Stimmen der Weltkirche, Nr. 41).

Tuazon R. A., Kulturelle Minderheiten und die katholische Soziallehre, „Concilium“ 53 (2017), S. 274-283.

Weber W., Der soziale Lehrauftrag der Kirche. Katholische Soziallehre in Text und Kommentar, Köln 1975.

Wiemeyer J., Die Kapitalismuskritik von Papst Franziskus, in: Der Kapitalismus - ein Feindbild für die Kirchen?, Hrsg. S. Wirz, Zürich-Baden-Baden 2018 (im Erscheinen).

Wiemeyer J., Evangelii Gaudium - das Programm eines Pontifikats, „Die neue Ordnung“ 68 (2014), S. 100-109.

Wiemeyer J., Keine Freiheit ohne Gerechtigkeit, Freiburg i Br. 2015.

Wiemeyer J., Papst Franziskus und die Wirtschaft, „Amosinternational Gesellschaft gerecht gestalten“ 8 (2014), S. 50-53. 